\title{
The hot stars in orbit around the M 31 central supermassive black hole: are they young or old?
}

\author{
P. Demarque and S. Virani
}

\author{
Department of Astronomy, Yale University, PO Box 208101, New Haven, CT 06520-8101, USA \\ e-mail: [pierre.demarque; shanil.virani]@yale.edu
}

Received 27 June 2006 / Accepted 2 October 2006

ABSTRACT

\begin{abstract}
Aims. The cluster of hot stars observed in orbit around the central black hole of M 31 has been interpreted as a 200 Myr starburst. The formation of a population of young stars in close proximity to a massive black hole presents a difficult challenge to star formation theory. We point out that in a high stellar density environment, the course of stellar evolution is modified by frequent collisions and mergers.

Methods. Blue stragglers, which are the results of mergers in globular clusters, occupy the same position in the color-magnitude diagram as the observed hot stars in M 31. For confirmation, the integrated spectrum of P3 is shown to be compatible with the spectral energy distribution of a blue horizontal branch field star.

Results. We suggest an old stellar population of evolved blue horizontal-branch stars and of merger products cannot be ruled out on the basis of the available data. Observations are suggested that would help distinguish between a "young" and "old" stellar population interpretation of the observations.
\end{abstract}

Key words. stars: evolution - galaxies: active - galaxies: individual: M31

\section{Introduction}

Recent observations of the central region of M 31 by Bender et al. (2005) have yielded convincing evidence for the presence of a central supermassive black hole in M31. M31 is thus the third example of a galaxy with a well determined central black hole $(\mathrm{BH})$ mass, after our Galaxy (Ghez et al. 2003, 2005; Genzel et al. 2003) and M32 (van der Marel \& van den Bosch 1998).

Bender et al. (2005) identified three distinct stellar populations near the center of M 31, denoted as P1, P2 and P3. P1 and P2 had been studied by previous authors (Lauer et al. 1993; King et al. 1995). Bender et al. (2005) gave the name P3 to the compact source embedded within P2, whose strength in the ultraviolet had first been noted by King et al. (1995). These authors discovered that the stellar population in P3 defined a disk like distribution orbiting the $\mathrm{M} 31$ central $\mathrm{BH}$, and were thus able to derive a mass of the order of $1.1-2.3 \times 10^{8} M_{\odot}$ for the massive $\mathrm{BH}$, based on the measured velocities of the orbiting stars.

From the spectrum, Bender et al. (2005), recognized the population substratum in $\mathrm{P} 1$ and $\mathrm{P} 2$ as an old stellar population. They identified the hot stars in P3, used in the central BH analysis, as young stars, and argued, with the help of stellar population models (Bruzual \& Charlot 2003; Aparicio \& Gallart 2004), that the spectrum is best interpreted as that of a $200 \mathrm{Myr}$ starburst.

Although the dynamical argument used in deriving the mass of the central $\mathrm{BH}$ is independent of the nature of the hot stars whose motions were measured, it is important to understand the evolutionary status of the stars in the central cusp of M 31 . The identification of these stars as young stars poses challenging astrophysical problems regarding their origin, and about the star formation process in the close vicinity of a massive
BH (Alexander 2005). It presents also dynamical difficulties if these stars were formed well away from the central $\mathrm{BH}$ and migrated towards it. The mediation of a second $\mathrm{BH}$, of intermediate mass (in the range $10^{3}-10^{4} M_{\odot}$ ) has been suggested (Hansen \& Milosavljevic 2003).

In this paper, we address the issue of the stellar population and age of the observed hot stars. We point out that a case can be made that the observed spectrum does not necessarily belong to a 200 Myr starburst. It could also belong to an old stellar population, in the post-HB evolutionary phase, or a population in which collisions have modified the course of stellar evolution. While the young starburst hypothesis may well turn out to be correct, we note on the basis of the available information alone, that we cannot rule out the possibility that the observed stellar population is old. The observed hot stars may be evolved stars that have lost most of their envelope, or that are the result of recent mergers in a dense environment. The normal course of stellar evolution is modified in high stellar density environments (Alexander 2005). The blue stragglers observed in globular star clusters are an example of such mergers (Bailyn 1995; Sills \& Bailyn 1999). Figure 1, borrowed from Sills \& Bailyn (1999), illustrates the position of blue stragglers in the color-magnitude diagram. The main point of this paper is to discuss this alternative interpretation, which if correct, would resolve some of the astrophysical difficulties associated with the young starburst hypothesis. The construction of models specifically applicable to M 31 that take account of stellar interactions and mergers in high density regions is beyond the scope of this paper but will be the topic of a future paper. Finally, we suggest observations that would help distinguish between the "old" and "young" stellar population interpretations.

This discussion would not be complete without recalling that a similar question can be asked on the formation process of the 


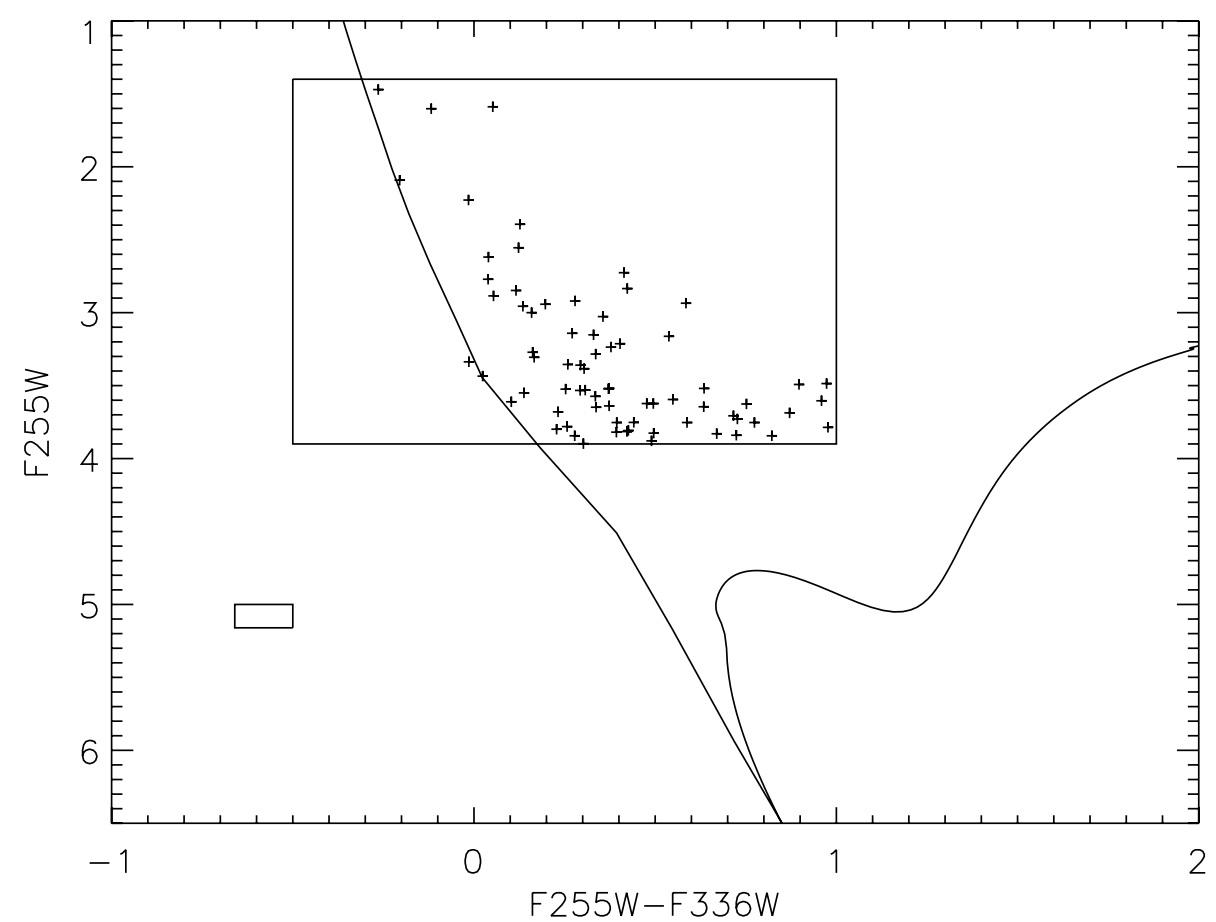

Fig. 1. Schematic color-magnitude diagram of a globular star cluster in the HST ultraviolet filters $F 255 W$ and $F 336 W$. The box indicates the position of observed blue straggler stars, believed to be the results of mergers. The position of the zero-age main sequence is marked by a solid line. The other solid line is the position of the fiducial stellar sequence of the cluster. The plotted blue straggler data are from Ferraro et al. (1997). This figure is taken from Sills \& Bailyn (1999, Fig. 3).

high velocity hot stars observed near the Galactic center, which are also usually believed to be very young. It is tempting to draw an analogy between the Galactic center stars and those observed at the center of M 31. We do this cautiously, however, fully aware that more detailed information is available on the environment of the hot stars observed in the Galactic center than in the case of M31. In addition, the Galactic center stars are B and $\mathrm{O}$ stars, much hotter than the A stars detected in M31, and it is possible that their origin may differ as well.

\section{The discovery of hot stars in orbit around the central black hole in M 31}

It is King et al. (1995) who discovered that P2 is much brighter than P1 in the ultra-violet. They also pointed out that there is a compact source embedded in P2, which was later called P3 by Bender et al. (2005). King et al. (1995) also noted that this compact source is similar in color and brightness to a single postasymptotic giant branch (PAGB) star. PAGB stars are observed in relatively old metal-rich stellar populations of the kind found in elliptical galaxies and galactic bulges. More than a decade ago, King et al. (1992) had found evidence from HST FOC data in the M31 bulge that half of the UV light came from PAGB stars. They attributed the rest of the observed UV background to evolved stars from a yet unresolved stellar population, possibly an old metal-rich population of the kind discussed by Horch et al. (1992) and Castellani et al. (1992). In a later study, King et al. (1995) favored a nonthermal light (AGN) interpretation, for the compact source in $\mathrm{P} 2$.

Further observations by Lauer et al. (1998) and Brown et al. (1998) ruled out the single PAGB explanation by resolving the strong UV source into a cluster of stars. Finally, combining the observed ultra-violet and optical fluxes, Lauer et al. (1998) concluded that the integrated light was consistent with a spectral energy distribution similar to that of an A star.

The A star interpretation was recently confirmed by Bender et al. (2005), who obtained the first integrated spectrum for the P3 peak embedded within P2 using STIS. These authors demonstrated that the spectrum matches quite closely the spectrum of an A0 giant or A0 dwarf. After subtracting the background light from the P2 stellar population, they then made a plausible case that the stars in P3 are a 200 Myr starburst. Although they were aware of the astrophysical difficulties posed by the existence of young stars so close to the M 31 central BH, they merely commented that this phenomenon seems to occur in nature, appealing to the presence of hot stars near the Galactic central $\mathrm{BH}$. The challenges to star formation theory and stellar dynamics near a massive $\mathrm{BH}$ have already given rise to an extensive literature in the context of the Galactic central BH (see Nayakshin \& Sunyaev 2005, and references therein, for additional discussion).

\section{Evidence in favor of the young stellar population interpretation}

\subsection{Modeling the stellar population in $P 3$}

Bender et al. (2005) have recently obtained the SED for P3 which matches a dwarf or giant A0 stellar spectrum. In fact, for all practical purposes, a clump of stars in the spectral range B5-A5, with an integrated magnitude $M_{v}=-5.7$, would reproduce the spectrum satisfactorily. Bender et al. (2005) have interpreted their observations as evidence for a starburst, which they have modeled using the web based population synthesis program of Bruzual \& Charlot (2003). The result favors an age of about $200 \mathrm{Myr}$, although the red end of the spectrum matches a somewhat older population, with age of $510 \mathrm{Myr}$. For illustration, they 
constructed a CMD for a 200 Myr stellar population of solar metallicity using the IAC-STAR web based population program (Aparicio \& Gallart 2004). Bender et al. (2005) scaled the model so that 186 main sequence stars on the spectral range B5-A5 dominate the integrated spectrum. Other parameters of the starbursts (IMF slope and mass range) are not specified; they are indeed irrelevant since only the B5-A5 stars are observable and the rest of the stellar population is not detectable, because it is either too faint or because it cannot be separated from the P2 stellar background. The IAC-STAR model is confirmed by synthetic CMDs constructed for a range of parameters using the web based population $Y^{2}$ synthesis program ${ }^{1}$ which is based on an independent grid of evolutionary tracks (Demarque et al. 2004). As emphasized above, any population model dominated by the light of A0 stars, and whose integrated magnitude $M_{v}=-5.7$, satisfies the observational constraint.

\subsubsection{A population of evolved stars}

Two comments must be made about the web based population models quoted above: (1) they do not include, or include only in a approximate way the advanced phases of evolution for older stars which are known to produce hot stars in the CMD; (2) most importantly in the context of M $31^{*}$, they describe systems of non-interacting stars. We know that interactions between stars modify stellar evolution for many stars in the central regions of globular clusters (Sills \& Bailyn 1999; Sills et al. 2002). Since in the vicinity of the central BH, the stellar densities can be two orders of magnitude higher than in a typical globular cluster central region (Alexander 2005), interactions between individual stars are extremely frequent.

In a realistic population modeling for $\mathrm{M} 31^{*}$, these two points must be taken into account. More parameters must be considered in the construction of synthetic models, such as the stellar densities and probabilities for envelope stripping and stellar mergers. Other key parameters including chemical enrichment (the ratio $\Delta Y / \Delta Z$ ), amount of mass transferred and heavy element content must also be considered, making the task of population synthesis more complex than simply producing a single CMD for non-interacting stars. In addition, the products of stellar mergers in collisions must be considered.

Relevant detailed stellar structure calculations and population models already exist, well documented in the literature, which justify the plausibility of our argument (e.g. Sills et al. 2002; Yi et al. 1997b). For the present purpose, specialized studies can be referred to, in particular Figs. 7 to 9 in Yi et al. (1997b). The construction of models specifically applicable to M 31 that take account of these effects is beyond the scope of this paper but will be the topic of a future paper.

\section{Evidence in favor for an old stellar population interpretation}

\subsection{Old hot stars and the UV upturn in elliptical galaxies}

It has now become generally accepted that the UV upturn in elliptical galaxies is due to blue horizontal-branch (BHB) and post-HB stars (Yi et al. 1999). These stars are found in old stellar systems. Although the UV upturn is attributed to the presence of B-type or hotter stars, only a modest variation in envelope mass and chemical composition is needed to create an HB morphology dominated by the light from A stars (Lee et al. 1994).

\footnotetext{
${ }^{1}$ http://www. astro.yale.edu/demarque/yyiso.html
}

It was originally argued that high metallicity (likely in galaxy bulges) favor the production of luminous blue stars (Horch et al. 1992; Bressan et al. 1994), but further work has shown that it is not necessarily the case (Dorman et al. 1995; D'Cruz et al. 1996; Yi et al. 1997a, 1999), and that several combinations of helium abundance and envelope mass could produce luminous blue stars. A recent review by Yi \& Yoon (2004) discusses the present status of the BHB interpretation for the UV upturn. Much better data have recently been obtained with the GALEX mission (e.g Rey et al. 2005); but there remains some uncertainties regarding the relative importance of metallicity, and the frequency of binary stars. In addition, as discussed below in this paper, the stripping of giant envelopes, and in some cases, stellar mergers could play an important role in regions of high stellar densities (Bailyn 1995; Sills \& Bailyn 1999; Alexander 1999).

Lee et al. (1994) showed that the difference in morphology between a HB whose integrated light is dominated by $\mathrm{B}$ and $\mathrm{O}$ stars and another $\mathrm{HB}$ whose integrated light is dominated by A stars depends solely on plausible differences in chemical composition, age and envelope mass loss. We do not know the chemical composition nor age of the P3 stars in M31. But we do have observational evidence for the existence of a population of evolved old stars in the bulge of M 31, including luminous blue stars.

In this paper, we propose that, within the uncertainties of current stellar evolution theory, the interpretation that P3 is made up of an evolved old stellar population, is a viable alternative to the young starburst interpretation. Our point is illustrated in Fig. 2, where we have juxtaposed the observed spectrum Bender et al. (2005) with the spectrum of an AOV star and the spectrum of a field BHB star from the Sloan Digital Sky Survey (Sirko et al. 2004).

The observed spectrum could be either similar to the hot stars responsible for the UV upturn observed in elliptical galaxies and in the bulge of M31, or the result of close interactions due to high stellar densities (Alexander 1999). Finding such stellar populations near the center of a galaxy, either as a result of past star formation within the bulge, or through accretion of a dwarf satellite galaxy, would not be surprising in the context of what we know about the M 31 bulge stellar populations and our current knowledge on galaxy evolution.

\subsection{Evidence from $X$-rays}

X-ray observations provide crucial additional information about the age of the stellar population. X-rays would be expected to radiate from the cool stars in a young starburst, due to their large magnetic activity. Young cool stars in star forming regions such as Orion have been observed as X-ray sources. The reported total hard X-ray luminosity $(2-8 \mathrm{keV})$ in the Orion nebula is $1.2 \times 10^{33} \mathrm{erg} \mathrm{s}^{-1}$ (Feigelson et al. 2005). Recently, Garcia et al. (2005) reported a $3 \sigma$ upper limit to the emitted luminosity at the location of M $31^{*}$ of $1.0 \times 10^{36} \mathrm{erg} \mathrm{s}^{-1}$. This luminosity, however, is based only 13 net counts and clearly needs to be confirmed. Nevertheless, even a confirmed X-ray detection of M $31^{*}$ does not necessarily imply that the stars in its immediate vicinity are young, as the detected X-rays are likely due to the presence of the AGN rather than the X-ray emission from young stars as in Orion. Given the current spatial resolution of the Chandra X-ray Observatory, it is not possible to separate the X-ray emission from a putative population of young stars near M $31^{*}$ and from M 31* itself (Generation-X should be able to do so, however). On the other hand, if subsequent observations do not detect X-ray emission from the center of M 31, this must mean that either the 

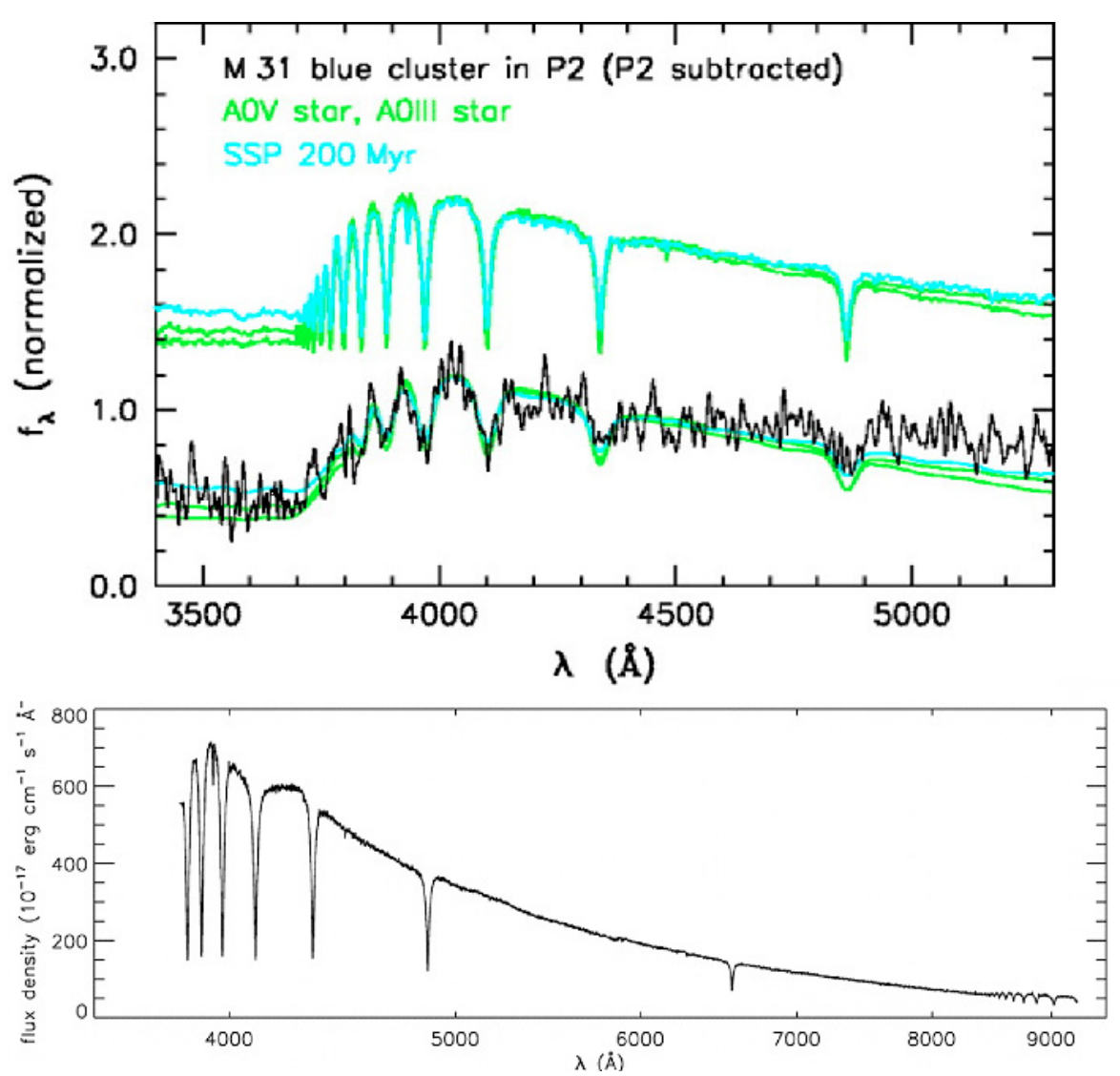

Fig. 2. The observed SED of P3 in M 31 (Bender et al. 2005) is the black curve shown in the top panel. It is compared to the SEDs of an A0 dwarf and giant, above (adapted from Bender et al. 2005, Fig. 4), and to the SED of a blue HB field star in the Galactic halo, below (from Fig. 2 of Sirko et al. 2004). At the available spectral resolution, either stellar SED could fit the observed SED.

cool stars present are weak X-ray emitters because the observed stellar population is old, or that there are too few cool stars in the population, perhaps suggesting an IMF differing from the canonical Salpeter law (Nayakshin \& Sunyaev 2005).

\subsection{Possible analogy with the center of our Galaxy}

Although the situation may be quite different in the Galaxy and in M31, we recall the similarities with the region of the central $\mathrm{BH}$ of our Galaxy. This similarity was appealed to by Bender et al. (2005) to explain the plausibility of observing young stars near the central BH. We draw this parallel tentatively because there are a number of differences between the observations in the Galaxy and M31. The high velocity hot stars observed in the Galaxy are B and O stars, markedly hotter than the A stars that dominate the M 31 spectrum. If they are old stars, because HB morphology is so sensitive to details of chemical composition and age, and to the binary fraction, in ways that are not fully understood, they need only differ in one or several of these characteristics.

Most importantly, the central cusp environment favors the stripping of red giant envelopes and the occurrence of stellar mergers. All these factors favor the formation of hot luminous stars in old stellar populations.

\subsubsection{The hot stars in the Galactic center}

High velocity stars with early spectral types $(\mathrm{O}$ and $\mathrm{B})$ have been observed near the center of the Galaxy, in close proximity to the central BH (Ghez et al. 2003, 2005; Genzel et al. 2003).
In the central region of the Galaxy, outside the cusp, Genzel et al. (2003) concluded, from the $K$-band luminosity function that the stellar population was relatively old (about $8 \mathrm{Gyr}$ ) and characterized by a red giant branch and a red clump of core helium burning stars. This underlying stellar population is characteristic of the central bulge of the Galaxy. Genzel et al. (2003) then contrasted the stellar K luminosity function within the central cusp. They state that the "cusp within $\leq 1.5^{\prime}$ of Sgr A* appears to have a featureless luminosity function, suggesting that old, low mass, HB/red clump stars are lacking". They further state "likewise there appears to be fewer late-type giants. The innermost cusp also contains a group of moderately bright, earlytype stars that are tightly bound to the BH."

The striking difference between the inner cusp is significant, and the underlying stellar population could be explained in several ways. The favored interpretation of most has been that the $\mathrm{BH}$ environment somehow favors star formation, and in particular the formation of massive stars (Nayakshin \& Sunyaev 2005), although Genzel et al. (2003) consider a possible merger interpretation for the OB stars within $0.04 \mathrm{pc}$ of the Galactic center, named the S-stars by Ghez et al. (1998). Another interpretation is that dynamical interactions in the old stellar population is entirely responsible for the cusp phenomenon. The higher stellar densities cause close interactions between stars. The frequency of envelope stripping on the red giant branch, and/or stellar mergers, would be enhanced. Some of the stripped giants would never reach core helium burning, others would evolve as blue $\mathrm{HB}$ and post-HB objects. In addition, the probability of mergers is enhanced. All these lead to the formation of a variety of luminous blue stars in A or hotter temperature range. The 
entire stellar population would then be old, but characterized by a deficiency of red giants and of red HB stars, exactly what is observed.

A final comment can be made about the spectral identification of individual stars in the Galactic center vicinity. We note that these objects are highly obscured and reddened by interstellar material. Often referred to as "HeI stars" because of their helium emission or absorption features, their spectra have been studied by a number of authors (Krabbe et al. 1995; Tamblyn et al. 1996; Blum et al. 1996; Eckart \& Genzel 1997; Paumard et al. 2001). The spectra show a variety of features, and can be divided into several classes. Paumard et al. (2001)'s discussion highlights the diversity of their spectra, which have given rise to differences in identification and classification between independent teams of researchers. Since most of the hot stars in a gas rich environment which have been studied spectroscopically in detail are young stars, the Galactic center spectra have been compared to those. Young hot stars are massive stars, found in open star clusters or associations, and accordingly, the observed "HeI stars" have been assigned large masses and young ages.

Stellar spectra by themselves do not contain any information about the ages of stars. Stellar spectra depend sensitively on temperature and chemical composition, and to some extent on surface gravity. In the absence of other information, such as physical environment, or the population to which they belong, it is difficult to recognize a young star from an old star simply on the basis of its temperature and luminosity. The identifications made so far were made simply by analogy with young starbursts that have been observed in other regions of the Galaxy, well removed from the Galactic center.

It is well recognized that if these stars are young, their origin is difficult to explain. In addition, the lack of X-rays from the Galactic center can only be explained by introducing a nonstandard IMF (Salpeter 1955; Miller \& Scalo 1979) as recently suggested by Nayakshin \& Sunyaev (2005). Before accepting this important implication for star formation, one needs also to definitively rule out the possibility that the hot stars observed near the Galactic central BH belong to an old stellar population.

\subsection{Dynamical studies}

The disruption of a captured massive old star cluster or dwarf galaxy into a disk gravitating about the central $\mathrm{BH}$ has been proposed as an interpretation. Monte Carlo simulations applied to a dense star cluster near the center of our own Galaxy (Gürkan \& Rasio 2005) seem compatible with such an interpretation, as long as the cluster is sufficiently massive $\left(10^{6} M_{\odot}\right)$. We note, however, that the dynamical models of Gürkan \& Rasio (2005), designed for young star clusters, would not be directly applicable in the case of the capture of an old group of stars. In a young system, a large range of masses along the main sequence coexist, with the largest masses up to about $50 M_{\odot}$. By contrast, the most luminous stars in the UV in an old stellar system have progenitors with masses of the order of one solar mass. By the time they have evolved to the blue horizontal branch (BHB) and beyond, stars have lost about half of their original mass. In the case of stellar mergers, the merger product would approximately double its mass. This fundamental difference in mass range would modify the gravitational segregation and selective evaporation in the infalling cluster.

The central $0.1 \mathrm{pc}$ around the central $\mathrm{BH}$ are characterized by high stellar densities (up to about $10^{8} M_{\odot}$ per cubic parsec), or two orders of magnitude higher than at the center of globular star clusters. Stellar collisions are therefore very frequent.
Two-body interactions lead to star segregation with the more massive stars being more centrally concentrated.

Observations indicate that the old stars are well relaxed in their velocity distribution while the hot stars are not. Separating the two stellar populations is the main problem in interpreting the velocity data. A population of old stars which have relatively recently lost much of their mass due to mass loss and stellar interactions (as is the case for old blue stars), would not be dynamically relaxed. Some stars could be kicked up to much higher velocities than their progenitors, particularly in binary systems.

The dynamical interaction would lead to an excess of randomly distributed high velocities, which is observed among the S-stars. Other evidence for unusually high velocities due to direct interaction with the $\mathrm{BH}$ is provided by the recent discovery of hypervelocity stars (HVS), described in the next section.

We have pointed out that the bulge of our Galaxy and M 31 are known to be primarily populated with old stars. In addition, the capture of old stellar systems by the Galactic halo has been extensively documented (Ibata et al. 2003; Duffau et al. 2006). Finding the remnants of captured old stellar populations in the inner part of the galactic bulge would not be surprising. For example, a concentration of A stars is expected in HB models for $Z=0.01$ and an age of several Gyr (e.g. see Fig. 8 in Yi et al. 1997b). Even without accounting for mass loss due to tidal interactions, this would not be unreasonable for a typical satellite dwarf galaxy population near a large spiral galaxy like M 31.

\subsubsection{Hypervelocity stars}

The plausibility of finding hot old stars near the Galactic central BH is shown by the recent discovery of hypervelocity stars (HVSs) in the Galaxy. Their existence lends support to the conclusion that at least some of the B stars in the vicinity of the Galactic center are old. HVSs are believed to achieve their extremely high velocities by dynamical ejection from a binary system while interacting with a massive black hole (Hills 1988). Five HVSs have so far been discovered (Brown et al. 2005, 2006; Hirsch et al. 2005; Edelmann et al. 2005). If Hills's interpretation is correct, hypervelocity stars provide some information on the stellar population in the vicinity of the massive black hole in the Galaxy. We note that two of the discovered HVSs (Hirsch et al. 2005; Edelmann et al. 2005) have been identified as likely blue HB ( $\mathrm{sdB}$ ) candidates. The other three are also B stars observed by Brown et al. $(2005,2006)$. They were discovered in a search for blue HB stars and could be either main sequence B stars or subdwarf B stars (sdB). We know that sdBs evolve into luminous hot stars (Yi et al. 1999).

\section{Suggested observational tests}

A crucial test of the "old stars" interpretation for the stellar population near the M 31 central BH could be made by detecting the presence of the less luminous red giants and main sequence stars that are the signature of an old stellar system. Spectral information in the red is needed. In particular, spectroscopic evidence for the presence of late spectral type giants and dwarfs in P3, or the lack thereof, would provide a crucial test of the old vs. young interpretation. At the present time, X-ray observations of M 31 are insufficiently sensitive, and lack the required spatial resolution to make any statement about the presence (or absence) in P3 of young magnetically active cool stars, but future space missions will make the observations possible. Another test of the presence of young stars would be the detection of infrared excesses due to the presence of circumstellar disks (Yudin 2000). 
In our Galaxy, ongoing searches for HVSs, and more detailed studies of their characteristics will provide further indications of the stellar population near the massive BH. At this point, the available data from the HVSs, admittedly very scant, show that at least a fraction of the stars close to the massive $\mathrm{BH}$ at the Galactic center are old HB stars.

\section{Summary}

In this paper, we propose that the hot stars observed near the central BH of M 31 could belong to an old stellar population, similar to the stellar population observed in the M 31 bulge. The unusual physical environment (high stellar densities) in the vicinity of the central BH is expected to modify the normal course of stellar evolution in several possible ways:

1. By the stripping of a part or of the whole envelope of a red giant envelope. The end result is the formation of a blue HB star, either a sdB or a sdO, depending on the amount of mass loss, rather that red clump stars. Further, blue HB stars evolve into hot luminous stars instead of becoming asymptotic giants.

2. Through stellar collisions resulting in mergers. Such merger products form a population of luminous hot stars analogous to the blue stragglers observed in globular star clusters.

An old stellar population in which collisions are frequent would be characterized by a defect of red horizontal branch stars (red clump stars) and luminous red giants when compared to a normal population of the same age and chemical composition. At the same time, it should exhibit a population of hot stars of various luminosities and colors. In addition, having suffered recent collisions, the space motions of the hot star population would differ from that of the background population, and in particular could exhibit unusually high space velocities. The high velocity S-stars observed within pc of the Galactic central BH may be examples of such stars. In the extreme case of binary systems interacting with the central $\mathrm{BH}$ itself, close interaction could give rise to hypervelocity stars.

The old star interpretation has several advantages over the young stellar population interpretation. It removes the problem of understanding star forming regions in situ near the massive $\mathrm{BH}$ in the center of M31 and the Galaxy. In the case of the Galaxy, it explains naturally the absence of strong X-ray sources without appealing to a non-standard IMF (Nayakshin \& Sunyaev 2005). Moreover, Jimenez et al. (2006) in a recent preprint suggest that irradiation of a stellar atmosphere by an AGN can make late type stars appear young. Therefore, the confluence of these several independent lines of investigation suggest that the stars in the immediate vicinity of M 31 * could be explained by a population of old stars.

Acknowledgements. We thank the referee for comments that have improved the paper. The authors are indebted to P. Coppi, R. B. Larson, T. Lauer, E. Murphy, J.-H. Woo and R. J. Zinn for helpful conversations on the stellar populations near the central BHs of M 31 and the Galaxy. We have also benefitted from useful correspondence from W. Brown, T. Kinman and T. Davidge. Special thanks are due to P. Natarajan for reading an early draft of the paper and for suggesting helpful revisions that have improved the manuscript.

This work was supported in part by NASA grant NAG5-13299.

\section{References}

Alexander, T. 1999, ApJ, 527, 835

Alexander, T. 2005, Phys. Rep., 419, 65

Aparicio, A., \& Gallart, C. 2004, AJ, 128, 1465

Bailyn, C. D. 1995, ARA\&A, 33, 133

Bender, R., Kormendy, J., Bower, G., et al. 2005, ApJ, 631, 280

Blum, R. D., Sellgren, K., \& DePoy, D. L. 1996, ApJ, 470, 864

Bressan, A., Chiosi, C., \& Fagotto, F. 1994, ApJS, 94, 63

Brown, T., Ferguson, H. C., Deharveng, J.-M., \& Jedrzejewski, R. I. 1998, ApJ, 508, 139

Brown, W. R., Geller, M. J., Kenyon, S. J., Kurtz, M. J., et al. 2005, ApJ, 622, L33

Brown, W. R., Geller, M. J., Kenyon, S. J., Kurtz, M. J., et al. 2006, ApJ, submitted [arXiv: astro-ph/0601580v1]

Bruzual, G., \& Charlot, S. 2003, MNRAS, 344, 1000

Castellani, M., Limongi, A., \& Tornambè, A. 1992, ApJ, 389, 227

D’Cruz, N. L., Dorman, B., Rood, R. T., \& O'Connell, R. 1996, ApJ, 466, 359

Demarque, P., Woo, J.-H., Kim, Y.-C., \& Yi, S. 2004, ApJS, 155, 667

Dorman, B., O'Connell, R., \& Rood, R. T. 1995, ApJ, 442, 105

Duffau, S., Zinn, R., Vivas, A. K., et al. 2006, ApJ, 636, 97

Eckart, A., \& Genzel, R. 1997, MNRAS, 284, 576

Edelmann, H., Napiwotzki, R., Heber, U., Christlieb, N., \& Reimers, D. 2005, ApJ, 634, L181

Feigelson, E. D. 2005, ApJS, 160, 379

Ferraro, F. R., Paltrinieri, B., Fusi Pecci, F., et al. 1997, A\&A, 324, 915

Garcia, M. R., Williams, B. F., Yuan, F., et al. 2005, ApJ, 632, 1042

Gehz, A. M., Klein, B. L., Morris, M., Becklin, E. E., et al. 1998, ApJ, 509, 678

Gehz, A. M., Duchêne, G., Matthews, K., et al. 2003, ApJ, 586, 127

Gehz, A. M., Salim, S., Hornstein, S. D., et al. 2005, ApJ, 620, 744

Genzel, R., Schödel, R., Ott, T., et al. 2003, ApJ, 594, 812

Gürkan, M. A., \& Rasio, F. A. 2005, ApJ, 628, 236

Hansen, B. M. S., \& Milosavljevic, M. 2003, ApJ, 593, L77

Hills, J. G. 1988, Nature, 331, 687

Hirsch, H. A., Heber, U., O'Toole, S. J., \& Bresolin, F. 2005, A\&A, 444, L61

Horch, E., Demarque, P., \& Pinsonneault, M. H. 1992, ApJ, 388, 53

Ibata, R., Irwin, M., Lewis, G., Ferguson, A. M. N., \& Tanvir, N., MNRAS, 340, L21

Jimenez, R., da Silva, J. P., Peng Oh, S., Jorgensen, U. G., Merritt, D., et al. 2006 [arXiv: astro-ph/0601527]

King, I. R. 1992, ApJ, 397, L35

King, I. R., Stanford, S. A., \& Crane, P. 1995, AJ, 109, 164

Krabbe, A., Genzel, R., Eckart, A., et al. 1995, ApJ, 447, L95

Lauer, T .R., Faber, S. M., Groth, E. J., et al. 1993, AJ, 106, 1436

Lauer, T. R., Faber, S. M., Ajhar, E. A., Grillmair, C. J., \& Scowen, P. A. 1998, AJ, 116, 2263

Lee, Y.-W., Demarque, P., \& Zinn, R. 1994, ApJ, 423, 248

Miller, G. E., \& Scalo, J. M. 1979, ApJS, 41, 513

Nayakshin, A., \& Sunyaev, R. 2005, MNRAS, 364, L23

Paumard, T., Maillard, J. P., Morris, M., \& Rigaut, F. 2001, A\&A, 366, 466

Rey, S.-C., Rich, R. M., Lee, Y.-W., et al. 2005, ApJ, 619, 119

Salpeter, E. E. 1955, ApJ, 121, 161

Sills, A., \& Bailyn, C. D. 1999, ApJ, 513, 428

Sills, A., Adams, A., Davies, M. B., \& Bate, M. R. 2002, MNRAS, 332, 49

Sirko, E., Goodman, J., \& Knapp, G. R. 2004, AJ, 127, 899

Tamblyn, P., Rieke, G. H., Hanson, M. M., et al. 1996, ApJ, 456, 194

van der Marel, R. P., \& van den Bosch, F. C. 1998, AJ, 116, 2220

Yi, S., \& Yoon, S.-J. 2004, Ap\&SS, 291, 201

Yi, S., Demarque, P., \& Kim, Y. -C. 1997a, ApJ, 482, 677

Yi, S., Demarque, P., \& Oemler, A. Jr. 1997b, ApJ, 486, 201

Yi, S., Lee, Y.-W., Woo, J.-H., et al. 1999, ApJ, 513, 128

Yudin, R. V. 2000, A\&AS, 144, 285 